\title{
Identifying engagement patterns with video annotation activities: A case study in professional development
}

\author{
Negin Mirriahi \\ University of South Australia \\ Jelena Jovanovic \\ University of Belgrade \\ Shane Dawson \\ University of South Australia \\ Dragan Gašević \\ University of Edinburgh
}

\author{
Abelardo Pardo \\ The University of Sydney
}

\begin{abstract}
The rapid growth of blended and online learning models in higher education has resulted in a parallel increase in the use of audio-visual resources among students and teachers. Despite the heavy adoption of video resources, there have been few studies investigating their effect on learning processes and even less so in the context of academic development. This paper uses learning analytic techniques to examine how academic teaching staff engage with a set of prescribed videos and video annotations in a professional development course. The data was collected from two offerings of the course at a large research-intensive university in Australia. The data was used to identify patterns of activity and transition states as users engaged with the course videos and video annotations. Latent class analysis and hidden Markov models were used to characterise the evolution of engagement throughout the course. The results provide a detailed description of the evolution of learner engagement that can be readily translated into action aimed at increasing the quality of the learning experience.
\end{abstract}

\section{Introduction}

With the rise in interest in blended learning, flipped classroom, and online learning approaches across the higher education sector there is a growing need for professional development of teaching staff. The inclusion of these technology rich environments places higher demands on teaching staff to revise their teaching approaches and develop new technological and pedagogical skills. While the adoption of online technologies is certainly an element of these new teaching approaches, the role of professional development programs should be to facilitate the effective integration of online technologies with student-centred leaning experiences (Torrisi-Steele \& Drew, 2013). Rather than focusing solely on developing the technological skills of academic teaching staff, their understanding of effective socio-constructive learning strategies will further allow them to adjust their lecturing role to that of a facilitator guiding students' knowledge and skill development. Hence, professional development programs should provide opportunities for teaching staff to develop their conceptions of learning and teaching, and guide them towards more facilitation (Owens, 2012). One such way to foster reflection and understanding of effective learning strategies alongside experiencing the use of new online technologies, is by integrating multimedia case-based learning providing opportunities to broaden awareness, views, and understanding (Koury et al., 2009). Case-based learning involves the provision of different learning and teaching related cases presented in videos typically followed by discussion and instructional guidance. This approach has been shown to develop analytical and reflective skills as learners are exposed to multiple perspectives (Goeze, Zottmann, Vogel, Fischer, \& Schrader, 2014). However, while online technologies and video case studies are beginning to be integrated into professional development programs, there is limited research on how academic teaching staff actually engage with a case study presented in the form of a video to aid their learning.

While prior research has often used self-reports to investigate the use of videos in the curriculum (Giannakos, 2013; Yousef, Chatti, \& Schroeder, 2014), more recent advances in analytics techniques afford 
new opportunities to capture objective and nuanced data from the learners' clickstream. The analysis of this behavioural data can provide insight into how learners engage with videos as part of their course learning activities (Brooks, Epp, Logan, \& Greer, 2011; Giannakos, Chorianopoulos, \& Chrisochoides, 2014, 2015). These forms of video analytics, can be extended beyond the clickstream data, such as video play, pause, forward, and rewind events, to include other associated learning activities (e.g., video annotations or invideo quizzes) (Mirriahi, Liaqat, Gašević, \& Dawson, 2016). The analysis of this data can assist in developing models for early identification of learners who are not engaged or undertaking less optimal learning pathways or study behaviour. Furthermore, user-engagement data can inform future changes to the learning design or provide grounds for actionable interventions. For example, Li, Kidziński, Jermann, and Dillenbourg (2015a, 2015b) analysed learners' interactions with course videos in the context of two MOOCs, with the aim to identify study strategies indicative of possible difficulties experienced by a learner in understanding a video.

Study strategies derived from trace data about learners' interaction with videos can be indicative of theoretical constructs such as cognitive load (Paas, Tuovinen, Tabbers, \& Van Gerven, 2003; van Gog \& Paas, 2012). Differences in study strategies can be between students who may have experienced a higher level of cognitive load and their peers who did not; for example, higher rewind and pause rates for students experiencing a higher cognitive load are observed by Li and colleagues (2015a, 2015b). Differences in study strategies are also found to be significantly associated with student performance (Li et al., 2015a, $2015 b)$. This suggests that the analysis of user trace data can potentially reduce the reliance on self-reported instruments as a measurement of cognitive and provide insights into (a) how study processes unfolded over time; and (b) what impact curriculum practices had on the study processes.

The study reported in this paper focuses on a professional development program using a case-based learning theoretical approach. The study used analytic techniques to examine how participants engaged with the video case studies and associated online activities to provide insight into their learning behaviour and adopted learning strategies, and contribute to the revision of future program offerings. The incorporation of learning analytic techniques affords an opportunity to examine how users navigate the course activities and how they apply specific technologies to aid their learning progress (e.g., Jeong, Biswas, Johnson, \& Howard, 2010; Lust, Elen, \& Clarebout, 2013a, 2013b).

\section{Self-regulated learning and learning strategies}

Professional development programs are lifelong learning experiences involving adult learners, and typically require their active engagement and self-regulation. This work is underpinned by the theory of selfregulated learning (SRL), and in particular by Winne and Hadwin's (1998) model of SRL. According to this model, learners are active agents who use cognitive, physical, and digital tools to operate on raw information in order to create products of learning. Learners regulate their learning by continuously evaluating the quality of their products and effectiveness of the chosen study tools and tactics. This process, known as metacognitive monitoring, is influenced by internal and external conditions. The former includes, a learner's level of motivation, prior knowledge, and affective states. The latter (i.e., external conditions) are determined by the elements of the instructional design (e.g., the teacher's role, course requirements, and availability of feedback). Learner's agency is also evident in the decisions the learner makes regarding the study tools and tactics they use to meet the requirements of the learning tasks.

According to Winne (2006), one of the key self-regulatory skills is the learner's capacity to choose a strategy that is optimal for the given internal and external conditions, and to adapt it as the conditions change. Learning strategy includes "any thoughts, behaviours, beliefs or emotions that facilitate the acquisition, understanding or later transfer of new knowledge and skills” (Weinstein, Husman, \& Dierking, 2000, p. 727). Hence, learning strategy is a latent construct that can neither be observed nor directly measured or assessed. Earlier research aimed at examining learning strategies mostly relied on learners' self-reports to acquire insights into strategies adopted by the learners. However, this data source suffers from deficiencies such as inaccuracy, due to the poor recall of prior behaviour (Winne \& Jamieson-Noel, 2002) or high level of cognitive load placed on the participants (Winne, 2013). When learning is done in digital environments, the data collected from the tools and services the students interact with during the learning process represent a better data source for studying learning strategies (Winne, 2013). Individual events logged in trace data can be considered proxies of primitive learning, whereas behavioural patterns (i.e., patterns of events) 
mined from the trace data can be considered as manifestations of the adopted study tactics and strategies (Winne, 2013).

The study presented in this paper examines learning strategies of teaching staff enrolled in a professional development program by relying on the user trace data collected from a video annotation tool used as the primary learning tool in the program. To extract learning strategies from the collected trace data, we relied on unsupervised statistical methods, such as clustering and hidden Markov models, which have proven beneficial for mining latent, unobservable constructs from learning traces (e.g., Blikstein et al., 2014; Kovanović, Gašević, Joksimović, Hatala, \& Adesope, 2015; Lust et al., 2013a).

\section{Engagement with course videos}

While few studies have investigated how teaching staff engage with instructional videos in professional development programs, there are numerous studies focusing on student engagement with videos and their associated activities (e.g., quizzes or annotations) in formal higher education courses. The findings indicate that there are identifiable patterns of user behaviour, indicative of students' learning strategies that can be used to inform teaching practice, support interventions, and course learning design. Most commonly, these analyses and their findings are used to identify groups of students with low or insufficient levels of engagement that may adversely impact their overall academic performance. For instance, Lust and colleagues (Lust et al., 2013b; Lust, Vandewaetere, Ceulemans, Elen, \& Clarebout, 2011) identified different students' strategies related to the use of various learning tools available within a learning management system (e.g., video recorded lectures and practice quizzes). In particular, based on the identified strategies, Lust et al. (2013b) recognised four groups of students: no-users, intensive users, selective users, and limited users.

Further, studies have examined the role of students' note taking (length and frequency) of lecture videos $(\mathrm{Mu}, 2010)$ and patterns of student engagement with lecture videos. For example, Brooks et al. (2011) identified five groups of students based on the detected patterns of student engagement with lecture videos: minimal active learners, highly active learners, deferred learners, and just-in-time learners. Similarly, Phillips, Maor, Presto, and Cumming-Potvin (2012) revealed ten engagement patterns. The groupings ranged from students who did not access the videos at all (non-users), those who only accessed the videos once (random and one-hit wonders), those who only accessed the videos 2 weeks prior to a final exam or assessment task (crammers), and those who accessed the videos weekly (conscientious). While these studies show how video analytics, can help reveal simple patterns in students' engagement that can promote the integration of learning support interventions or provide insight in the course design, other studies have specifically explored the use of video annotations as a pedagogical approach.

Various video annotation software have been integrated into teaching practice (e.g., Mirriahi \& Dawson, 2013). One of the earliest video annotation tools, the Microsoft Research Annotation System (MRAS), enabled students to make notes (e.g., time-stamped annotations) on particular sections of a video they perceived useful compared to traditional note-taking in live lectures (Bargeron, Gupta, Grudin, \& Sanocki, 1999). Other annotation tools, such as the Media Annotation Tool (MAT) had similar features to MRAS but with the additional functionality of annotating any type of media and sharing annotations with peers and teachers leading to more collaborative reflective learning. This tool has been previously used in academic professional development (Colasante, 2011). Feedback from students revealed their appreciation of peer and teacher feedback and an enhancement of their overall learning. However, the data used for the study was largely based on self-reported information collected via pre and post surveys and interviews, albeit also included direct observations of student and teacher interactions with MAT. Recent studies (Gašević, Mirriahi, \& Dawson, 2014; Pardo et al., 2015) have used video analytics techniques to analyse detailed event streams and investigate how first-year university students use and engage with video annotation tools.

The study described in this paper extends the current state of the art in learners' study strategies by applying learning analytic techniques on data captured by a video annotation tool in the context of a professional development program. In addition, unlike the majority of reported studies on student learning strategies that consider learning strategies as fixed constructs (i.e., once adopted stay the same throughout the course), we argue that learners change and adapt their strategies. In particular, as per our theoretical model, grounded in the Winne and Hadwin's (1998) model of SRL, we posit that learners tend to change their study tactics 
and strategies as a consequence of the changes in the external and internal conditions. Given that we are interested in lifelong learners and their professional development activities, different external pressures (e.g., those associated with their job) may force them to change learning strategies from one week to the next. Therefore, in this paper, we aim to study how learning strategies - derived from the collected trace data - change from one study unit to the next to account for the potential changes in external and/or internal conditions of the learners. In addition, we show that identification of study strategies, and especially their changes over time can offer insights into the effectiveness of the deployed learning design and indicate changes that might be required.

\section{Research design}

The study draws on user data generated from interaction with course videos and associated annotations to identify how teaching staff in a higher education institution engage with video case-studies in a professional development course. More precisely, relying on the theoretical framework by Lockyer, Heathcote, and Dawson (2013) that connects learning analytics and learning design, the study explores types of user engagement patterns (indicative of users' learning strategies) that could inform subsequent revisions of the course design. Additionally, the study examines variation in engagement patterns over time in order to determine how often and in what way teaching staff change their learning strategies related to video case studies, so that program coordinators can anticipate such changes and provide appropriate support. Aiming to ensure high effectiveness of the learning experience, the presented study seeks to develop a model that can predict an individual's level of engagement at different points during the program.

\section{Research questions}

The study seeks to answer the following research questions:

1. What kinds of study strategies related to the use and annotation of the course videos can be detected in a case-based professional development program?

2. Can we detect common trajectories of learning strategies; that is groups of learners that may change their learning strategies during the program in a similar way?

3. Can a model be developed to predict a learner's trajectory of learning strategies within the program?

\section{Context}

This study took place at a large research-intensive higher education institution in Australia. The institution provides a non-accredited professional development program for its teaching staff to learn about effective learning and teaching strategies, reflect on them, and consider how they may apply them to their own teaching contexts. Program participants nominate themselves for enrolment. Participants are typically early career academics, but the program also includes more experienced teaching staff as well. The program is offered through blended delivery each semester and comprises of five modules each of which includes a combination of online activities and one face-to-face session. The intended learning outcomes of the professional development program were for teaching staff to be able to review their teaching and learning practice by drawing on various sources of evidence and subsequently plan effective activities and assessments. Program participants demonstrated achievement of these learning outcomes by completing a reflective e-portfolio. The learning activity central to this study involved a series of video case studies discussing various teaching strategies and issues that teaching staff commonly face.

The use of the open source Online Video Annotation for Learning (OVAL) software was piloted in the program in semesters 1 and 2 in 2015 to raise awareness of different online learning strategies and to prompt staff to reflect on their teaching strategies. OVAL was developed from the Collaborative Lecture Annotation System.

Teaching staff were requested to watch the video case studies using the OVAL software and post their individual annotations on the particular segments of the video that they related to, or considered interesting. Such annotations, tied to particular video segments, are referred to here as time-stamped annotations. Participants were then asked to review their time-stamped annotations and make a general annotation (nontime stamped) sharing their overall reflection on the video. All participants could view their peers' time 
and general annotations as well as their own. The enrolled teaching staff had further opportunity to discuss their view on the topics explored in the video case studies when they attended the face-to-face sessions. For the first module, the video annotation activity was a post-activity that they were asked to complete after the face-to-face session. However, for the remaining four modules, the video annotation activity was a preactivity whereby the enrolled students were encouraged to complete the activity prior to attending the faceto-face session where the video and their reflections on it were discussed.

\section{Sample}

The study included teaching staff enrolled in two offerings of the professional development program in Semesters 1 and 2 of 2015. In Semester 1 (S1), 77 teaching staff enrolled in the program and 68 of them used the video annotation tool. In Semester 2 (S2), 86 teaching staff enrolled in the program and 59 of them used the tool. The teaching staff members were at various levels of their careers and from a range of disciplines within the institution.

\section{Data collection and analysis methods}

Following institutional ethics approval, the data for the study was obtained from de-identified user logs from the OVAL video annotation tool. The analysis method consisted of three stages, each building on the previous, and thus gradually providing a deeper and more detailed insight into the learners' engagement patterns and study strategies. The method was applied to both offerings of the program in 2015, referred to hereafter as S1 and S2.

The first stage aimed to address the first research question. Here we applied a hierarchical clustering algorithm to the log data from the users' engagement with the OVAL video annotation tool in each module of the program. This enabled us to identify groups (clusters) of teaching staff with similar engagement patterns (i.e., learning strategies) in each module. Having repeated the clustering procedure with the log data from each of the program modules, we obtained for each user, a sequence of module-level cluster assignments. At the end of this data analysis stage, each user was described with a vector of five numerical values, each one representing the cluster assignment in the corresponding program module (i.e., identifying the user's learning strategy in the respective module). These vectors served as the input for the next data analysis step. For example, for a discrete user, we refer to here as Tom, this clustering step would produce the following numerical sequence for the period S1: $(1,2,3,3,4)$. This means that, based on the features of Tom's learning strategy in Module 1, he was assigned to Cluster 1, which after inspection of the strategy features we labelled active; in Module 2, he was assigned to Cluster 2 (satisfying module requirements) and so on.

The second stage of our analysis was focused on the second question and involved identifying common trajectories of learning strategies within the program, that is, the subpopulations of teaching staff with similar sequences of learning strategies identified in the clustering stage. To this end, we used latent class analysis (LCA). The algorithm identified subpopulations of teaching staff whose learning strategies related to the use of the videos and video annotation tool changed in a similar manner over the five modules of the program. We refer to the LCA results as trajectories (of learning strategies) since each one represents a trajectory or path of a group of learners who exhibited similar dynamics in their engagement patterns (i.e., learning strategies) within the program. Continuing with our example, based on this sequence of study strategies in S1 (active, satisfying module requirements, active, highly active, minimalist), user Tom would be assigned to Trajectory 1 (Figure 3), a trajectory common to a small group of users who stayed (more or less) active throughout the program.

Although LCA grouped teaching staff based on similar trajectories, the results did not show how likely a user was to preserve or change his/her strategy from one module to the next. To be able to predict changes in the users' learning strategies, and thus answer the third research question, the final data analysis stage consisted of modelling users' progress though the course as a hidden Markov model (HMM). In each module, a user exhibits a particular pattern of engagement with the OVAL tool which is modelled as a state in the HMM terminology. We expected that these states would correspond to the learning strategies identified in the clustering step. For example, we expected that one state might have been characterised by the engagement with the module videos and the annotation tool, but not beyond the level of the module requirements, thus corresponding to the satisfying module requirements strategy (identified in the clustering 
step). Even though HMM is based on the assumption that the next state depends only on the immediate previous state, which in the context of learning is not entirely correct, it has been shown that this statistical simplification leads to models that capture meaningful patterns and offer predictions of student behaviour (e.g., Blikstein et al., 2014; Jeong et al., 2010). The HMM provides a transition matrix with the probabilities of the transitions among states (including continued stay in the same state), so that in each module and for each user we can estimate how likely it is that they will keep the same or change their learning strategy. For example, if our user Tom is in the active state (i.e., learning strategy) and the transition matrix indicates that there is 0.33 chance of preserving that state and the probabilities to transitioning to other states (i.e., changing strategies) are significantly lower (Figure 4), we can expect that Tom will stay active in the next program's module, as well.

The following subsections provide a detailed description of the analytical methods undertaken in each of the noted three stages of analysis.

\section{Clustering}

In order to identify learners' engagement patterns with videos and the video annotation tool, the following set of features indicative of students' engagement (Mirriahi et al., 2016) were computed for each user and each program module:

- $\quad$ Features related to primitive learning operations (Winne \& Hadwin, 1998):

- $\quad$ Fast-forward: number of video forwarding events.

- Rewind: number of video rewind events.

- Non-stop: number of times the videos were played entirely without transitioning to another activity.

- Pause: number of times the videos were paused.

- Time watched: time videos were played (in seconds).

- General annotations total: number of general annotations added to the videos.

- Time-stamped annotations total: number of time-stamped annotations added to the videos.

- Annotations edited: number of edit operations on the video annotations (either general or time-stamped).

- Annotations deleted: number of annotations deleted.

- Features indicative of the overall coverage in student engagement (i.e., to what extent they were active in all activities planned in the learning design):

- Videos with general annotations: number of videos with at least one general annotation.

- Videos with time-stamped annotations: number of videos with at least one time-stamped annotation.

- Features related to time-management, as time management is one of the key issues in SRL and important to consider when studying learning strategies (Zimmerman, 2008):

- Earliest annotation added: length of time interval between the video being available and the first annotation (averaged across all videos, expressed in hours).

- Latest annotation added: length of time interval between the video being available and the time of the last annotation (averaged across all the videos, expressed in hours).

- Density of the transition graph: a transition graph was created for each participant, based on an adjacency matrix in which rows and columns were the types of events logged by the video annotation tool (value of element aij represents the frequency of event type i being followed by event type $\mathrm{j}$ ). The density of the resulting transition graph reflects the extent of the users' experimentation with different learning strategies, and thus, his/her level of metacognitive monitoring activity (Hadwin, Nesbit, Jamieson-Noel, Code, \& Winne, 2007).

The grouping of users with similar patterns was done using Ward's (1963) hierarchical clustering method with the normalised variables. 
Latent class analysis

Latent class analysis (LCA) is a subset of structural equation modelling that allows for finding groups or subtypes of cases in multivariate data (McCutcheon, 1987). It has been widely used to establish a typology of practices, profiles, and characteristics of individuals or phenomena. Having identified groups of teaching staff sharing common patterns of engagement within a module, the next step was to look for patterns in their engagement throughout the whole program. We identified three to five module-level clusters indicative of the study strategies adopted by each teaching staff participant. Each individual was associated with one cluster per course module, and henceforth a sequence of cluster assignments for the entire program. Since the professional development program consisted of five modules, the obtained sequences consisted of five nominal variables, each one representing the cluster the individual was assigned to in each module. These sequences were used as feature vectors for building the LCA model.

To decide on the number of clusters we relied on statistical information criteria, namely Akaike information criteria (AIC) (Akaike, 1973) and Bayesian information criteria (BIC) (Schwartz, 1978). Although there are no strict evaluation guidelines, LCA models that minimise the values of AIC and/or BIC, and with the Log Likelihood value closer to zero indicate a better fit, provided that the model has a good theoretical interpretation. LCA was performed using the PoLCA package (Linzer \& Lewis, 2011) of the R statistical language.

\section{Hidden Markov models}

The feature set used for building the HMM consisted of nine categorical variables derived from the features used for clustering. The number of states is estimated based on the number of recurring module-level clusters identified in the first stage of data analysis. The HMM model was built using the depmixS4 package (Visser \& Speekenbrink, 2010) of the $R$ statistical language.

\section{Results}

\section{Module-level learning strategies - Research question 1}

The clustering algorithm grouped teaching staff based on their patterns of engagement with the tool in each module of the program. The algorithm was applied to both offerings of the program (S1 and S2). Depending on the module, the results included either 3 or 4 groups of teaching staff exhibiting common engagement patterns indicative of the adopted learning strategies. Figure 1 shows the result for S1 and Figure 2 the result for S2. In both figures, each row corresponds to one module and shows the identified learning strategies and their level of presence in the corresponding module, that is, the percentage of the teaching staff with that strategy in the given module.

The identified strategies are:

- Active - This strategy is characterised by high level of engagement with the videos and the video annotation tool, and high number of authored/edited general and time-stamped annotations. The user who adopted this strategy were typically the first to annotate. The label is applied in the same sense as intensive users, those who made an effort to engage with all learning tools frequently and in an intensive manner (Lust et al., 2011), cyber cartographers who were goal-oriented and demonstrated self-efficacy (Barab, Bowdish, \& Lawless, 1997), highly active learners (Brooks et al., 2011), or conscientious learners who engaged with lecture video regularly on a weekly basis (Phillips et al., 2012).

- $\quad$ Satisfying module requirements - The group with this strategy was engaged with the module videos and the annotation tool, but not beyond the level of the module requirements. The label for this strategy is based largely on get it done approach users (del Valle \& Duffy, 2009), selective users who used technology strategically and were goal-oriented (Lust et al., 2011), or dominators who demonstrated focus and were goal-oriented (Cleave, Edelson, \& Beckwith, 1993).

- $\quad$ Catching up with the program requirements - This strategy is characterised by a moderate level of engagement with videos and annotations, and by engagement with videos of not only the current module, but also those of the previous modules, and doing it for the first time. This label is based 
loosely on deferred learners who accessed video lectures only towards the second half of the semester rather than regularly from the beginning (Brooks et al., 2011).

- $\quad$ Minimalists - The strategy shows marginal level of engagement with the video and low number or non-existing annotations. The label is based loosely on the term described by del Valle and Duffy (2009), and the minimal active learners described by Brooks et al. (2011).

- Disengaged - This label is used for users who did not interact with the videos nor the video annotation tool. The label is adapted from the no-users category proposed by Lust et al. (2013b) or non-users who did not engage with lecture video recordings (Phillips et al., 2012).

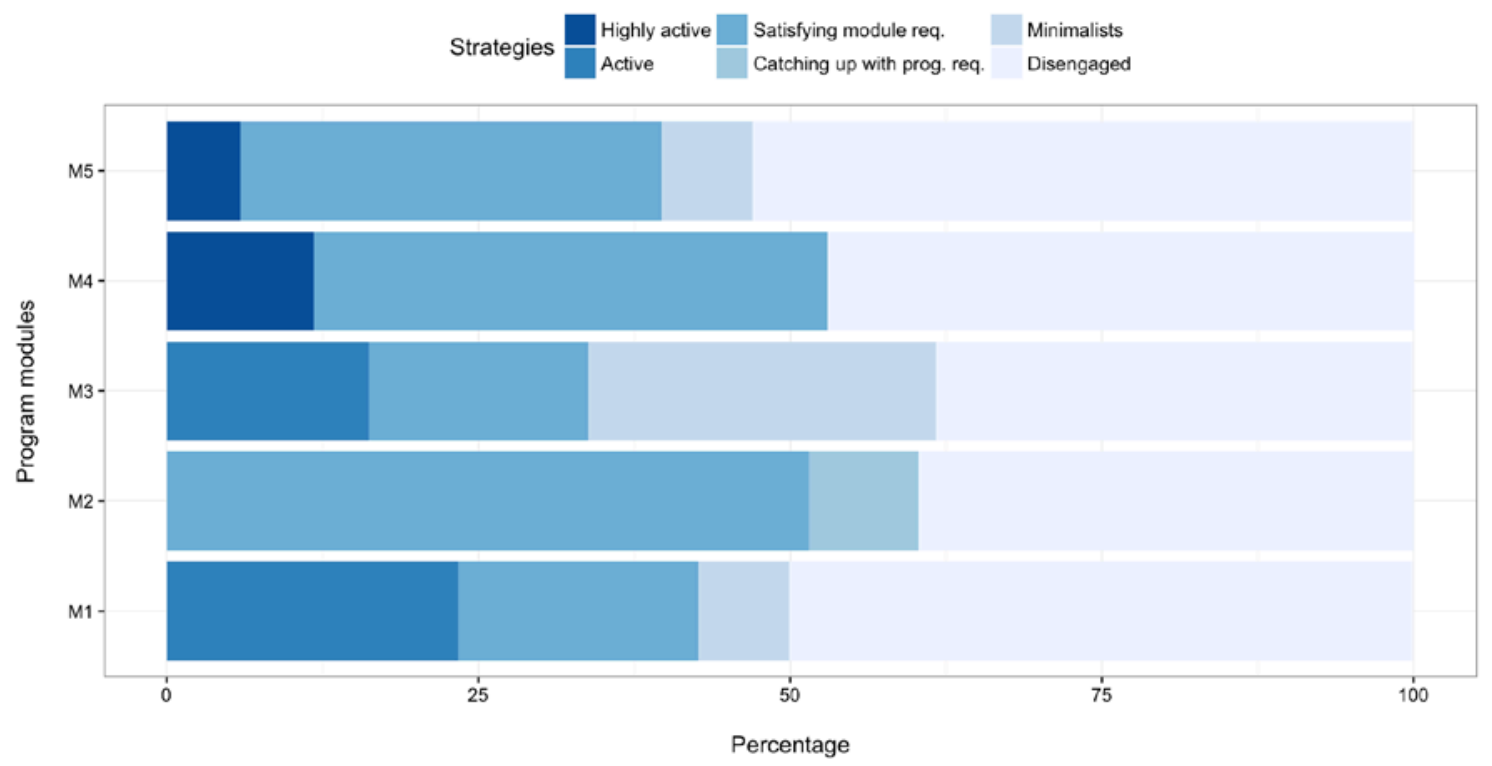

Figure 1. Percentage of staff adopting learning strategies for each module during the S1 offering.

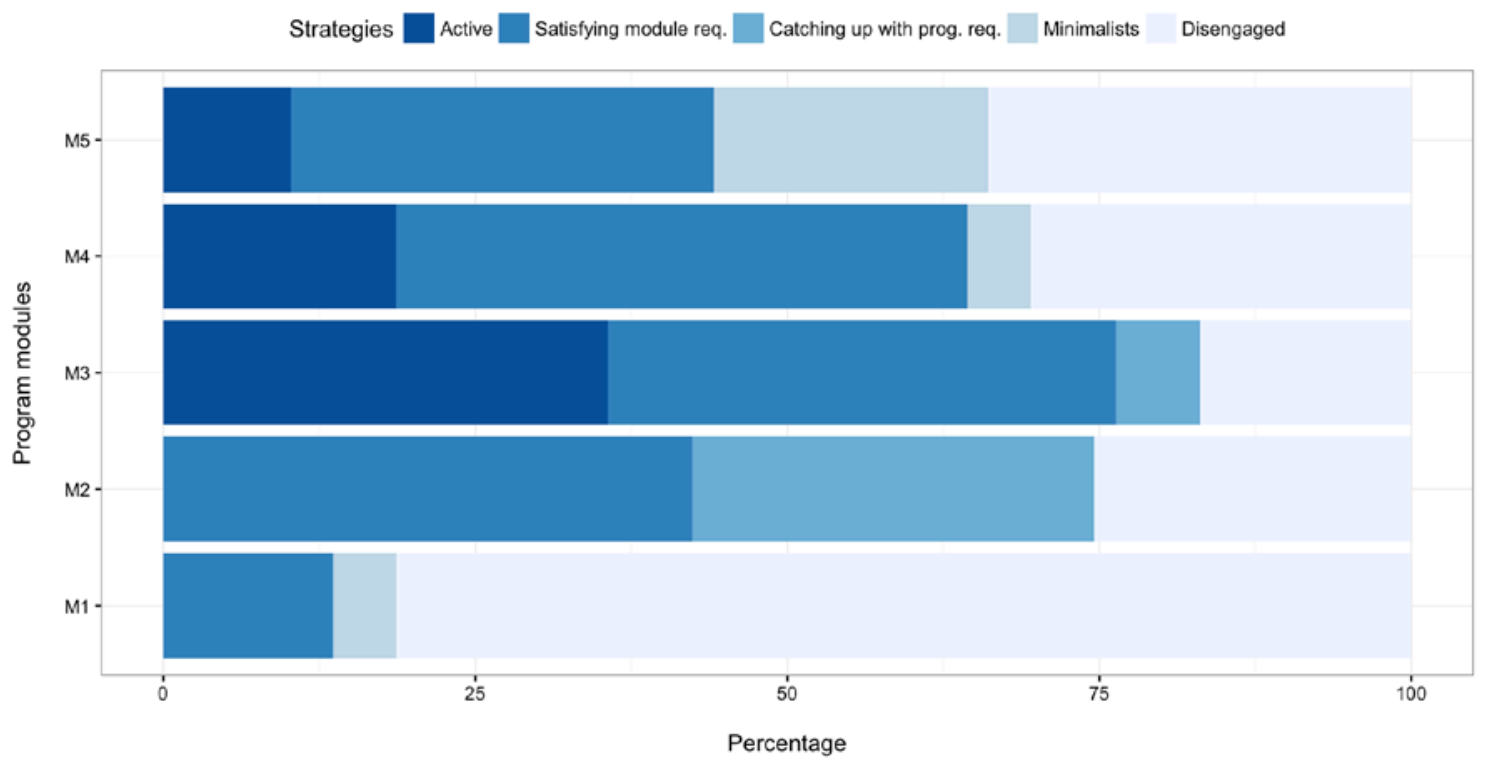

Figure 2. Percentage of staff adopting learning strategies for each module during the S2 offering.

The results show that modules have teaching staff with almost all kinds of identified learning strategies in both offerings. The only exception is the group with exceptionally high level of engagement - highly active strategy - which is present only in two modules of the S1 offering. 


\section{Program-level trajectories of learning strategies - Research question 2}

The evaluation metrics used to assess the LCA models are outlined in Table 1. We examined models with up to 4 classes. For the given sample size, models with more than 4 classes could not be built (the sample size was insufficient for the estimation of the model parameters). For the S1 offering, the metrics were not conclusive - the AIC metric suggested a 3-class model whereas BIC pointed a 2-class one. The model with 3 classes was chosen because it provided a more meaningful interpretation. This model is depicted in Figure 3.

Table 1

Metrics for LCA models with different number of classes (nclass) for S1 and S2 offerings

\begin{tabular}{c|c|c|c|c|c|c}
\hline \multirow{2}{*}{ nclass } & \multicolumn{3}{|c|}{ S1 offering } & \multicolumn{3}{c}{ S2 offering } \\
\cline { 2 - 7 } & AIC & BIC & LogLikelihood & AIC & BIC & LogLikelihood \\
\hline 1 & 831.76 & 865.05 & -400.88 & 659.39 & 686.40 & -316.70 \\
2 & 781.05 & $\mathbf{8 4 9 . 8 6}$ & -359.52 & 639.11 & 695.21 & -292.56 \\
3 & $\mathbf{7 7 4 . 1 3}$ & 878.45 & -340.06 & $\mathbf{6 3 7 . 2 3}$ & 722.41 & -277.61 \\
4 & 775.52 & 915.35 & -324.76 & 639.70 & 753.96 & -264.85 \\
\hline
\end{tabular}

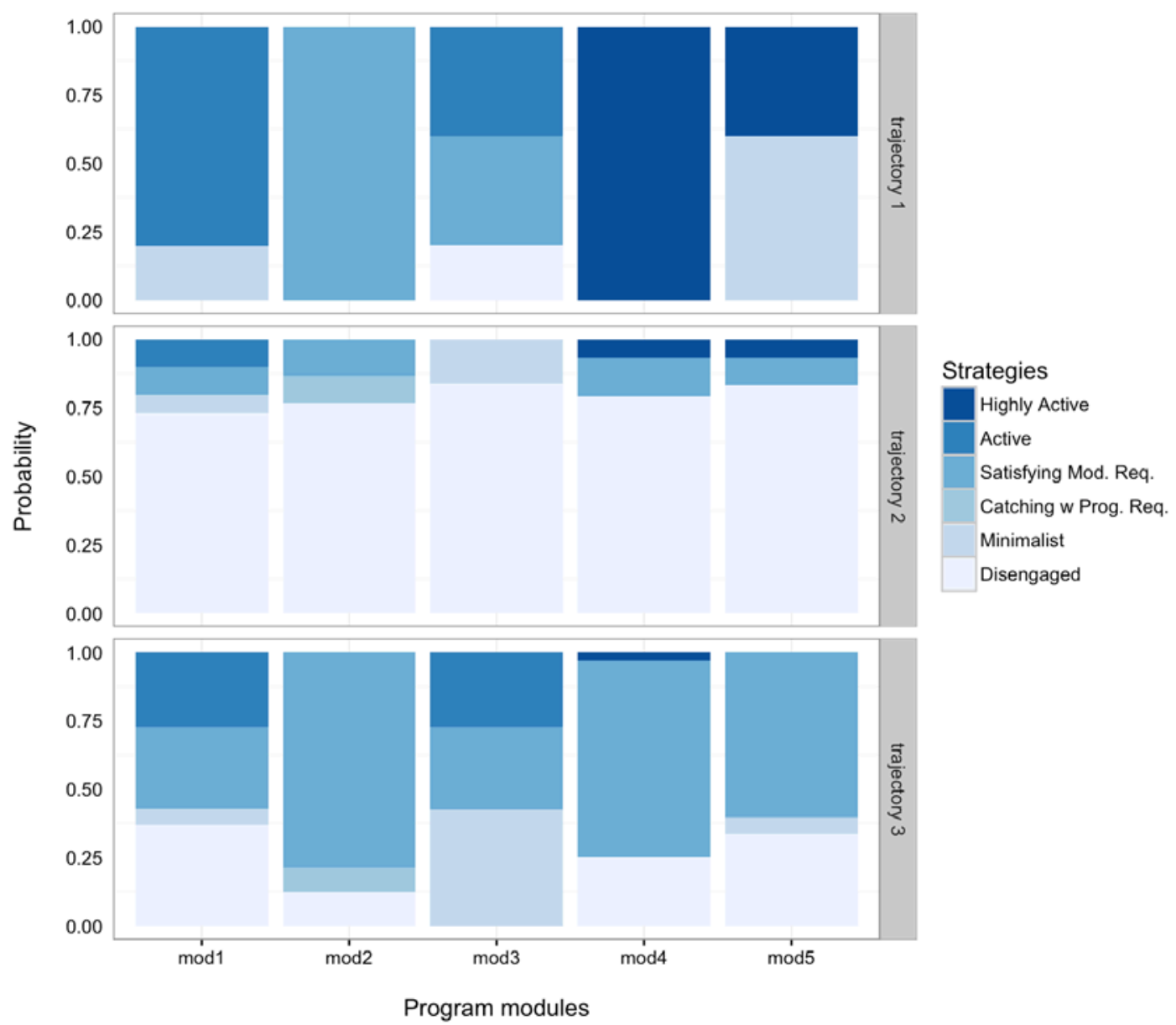

Figure 3. Trajectories of learning strategies obtained for the S1 offering. For each trajectory, the figure displays probability distribution for the six learning strategies.

For the S2 offering, the AIC metric identified the model with three classes (i.e., 3 trajectories) as the best one. The model for S2 is depicted on Figure 4. Figures 3 and 4 provide a visual representation of the trajectories adopted by the learners and how they evolve over time. 


\section{Model of strategy dynamics during the program - Research question 3}

The HMMs were calculated to provide insight into the dynamics of engagement with the videos and annotation during the program. A model was built for each of the two offerings. The number of states (ns) for the models was estimated based on the number of recurring module-level learning strategies identified in the first stage of the data analysis. Thus, we assumed the existence of five states $(n s=5)$ in each model, but also built models with $n s \pm 1$ states. After examining the resulting models for different $n s$ values, the models with five states were chosen for both offerings.

An HMM model provides, for each state, the estimated values for the feature set used. The examination of these values allowed to assign labels to the states of the resulting HMMs. Since the states correspond to the manifestations of the learners' strategies (as per our theoretical model), hereafter we refer to those states as learning strategies.

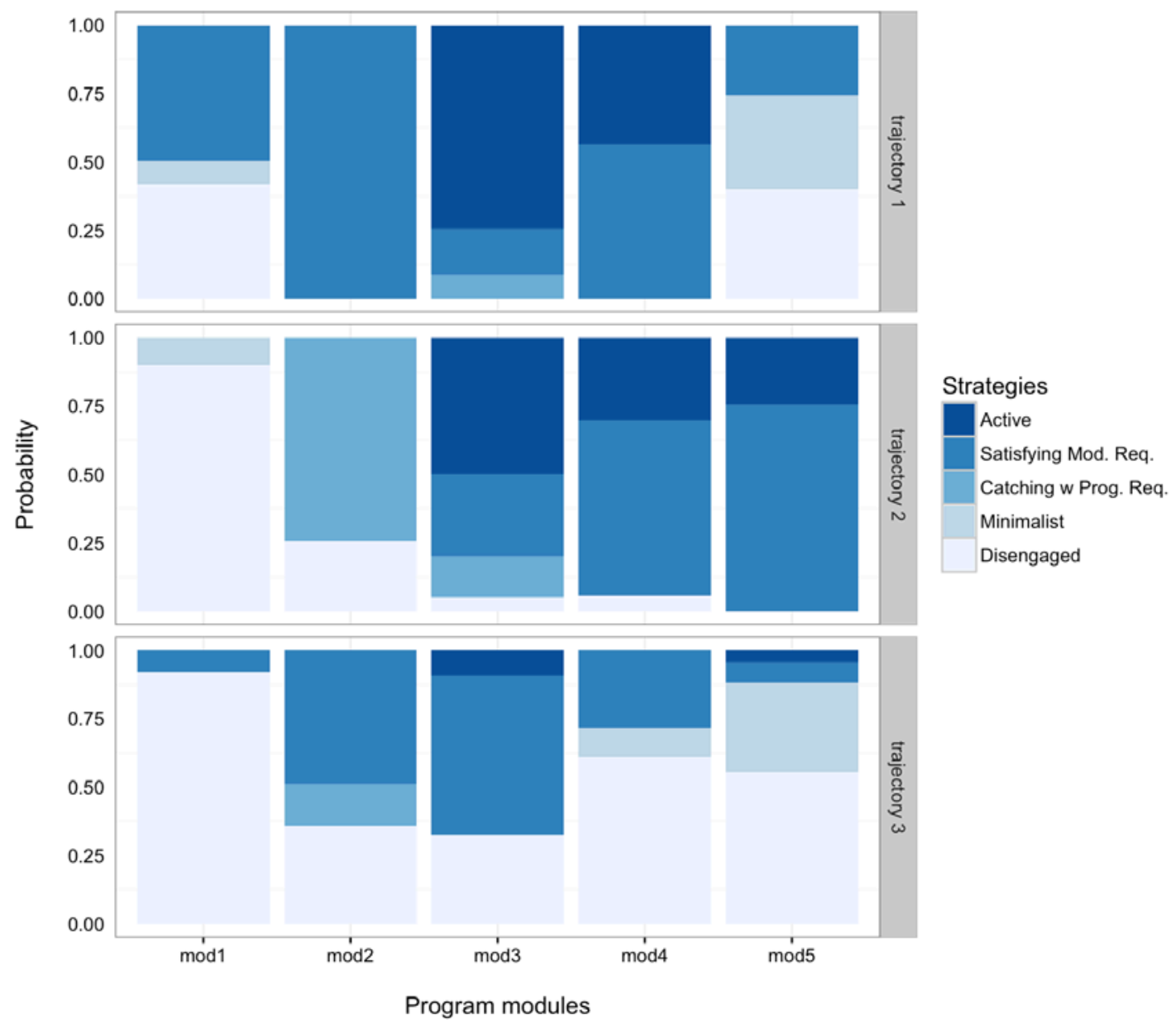

Figure 4. Trajectories of learning strategies obtained for the S2 offering. For each trajectory, the figure displays probability distribution for the six strategies.

As we assumed, the learning strategies identified with HMMs corresponded to the strategies identified in the first stage of our data analysis method. In particular, we identified four common strategies shared by S1 and S2 offerings: active, satisfying module requirements, minimalists, and disengaged. The strategy specific to the S1 offering is characterised by the learning behaviour of teaching staff that is somewhere between being completely disengaged and minimally engaged, and thus is labelled disengaged/minimalists. The strategy specific to the S2 offering is about the engagement pattern that we already encountered in the clustering step and labelled as catching up with the program requirements. For both offerings, the strategy with the highest probability to be the initial one is the one labelled as disengaged. 
Figure 5 depicts, for each module of the S1 program offering, the number of teaching staff per learning strategy identified by the HMM (each individual is associated with his/her most probable strategy in the given module). The figure show that the percentage of learners with the strategy satisfying module requirements stayed quite stable throughout the semester.

Figure 6 illustrates the distribution of individuals' most probable learning strategies in each module of the S2 offering. Clearly, a large majority of teaching staff (45 out of 59) exhibited complete lack of engagement during the first module. This changed significantly in the subsequent module where many of those previously disengaged, took a more active role and adopted one of the other four strategies: minimalists (14), active (9), satisfying module requirements (5), or catching up with the program requirements (5). During the modules 2, 3, and 4, the distribution of the teaching staff across the strategies stayed rather stable, to change only in the last module when primarily some of the active teaching staff transitioned to a lower pattern of engagement (minimalists or disengaged).

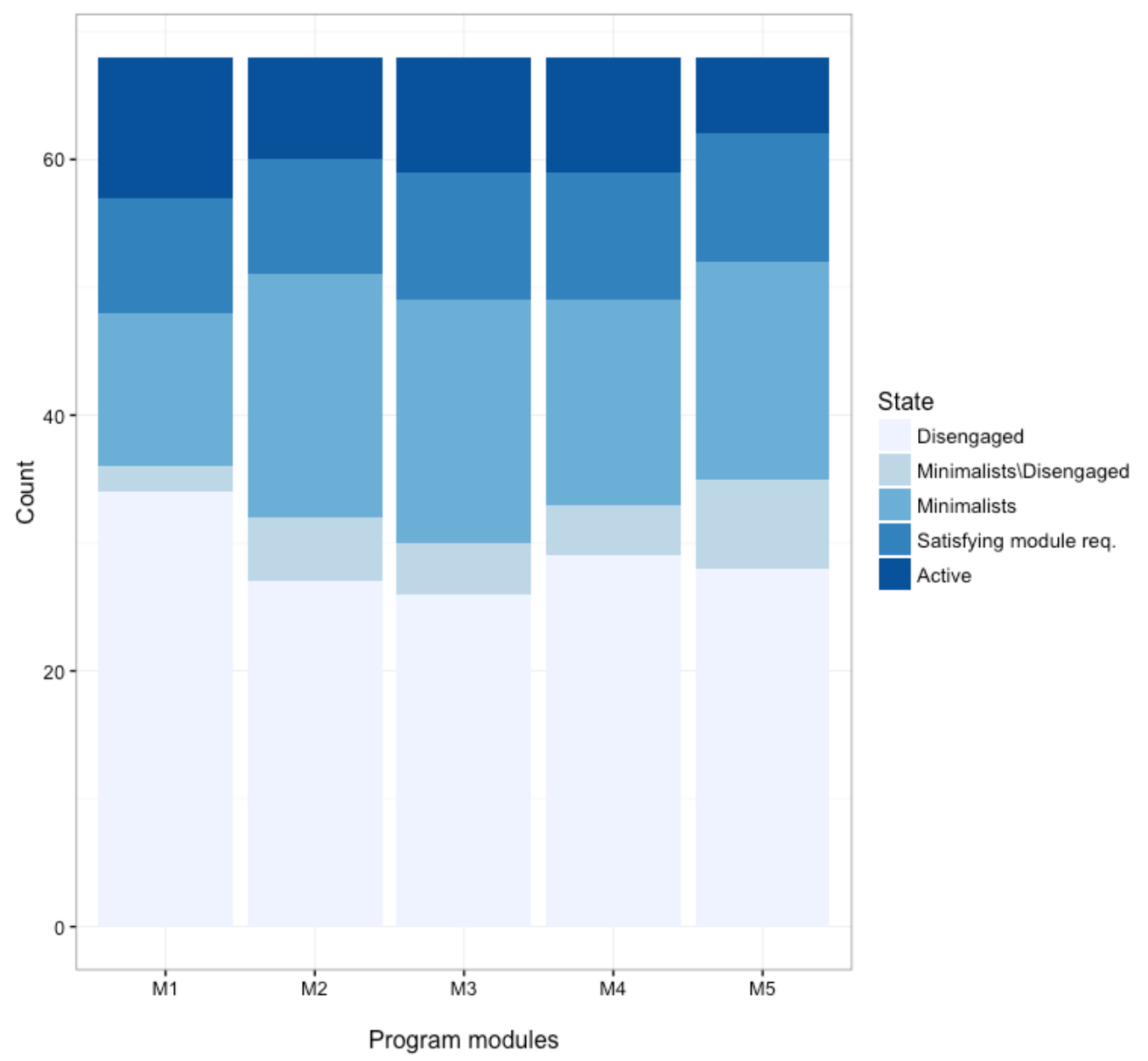

Figure 5. Distribution of teaching staff across the most probable learning strategies in modules (M1-M5), S1 offering.

\section{Discussion}

The results provide an increasing level of insight into the patterns of engagement (i.e., manifestations of the learning strategies) of the teaching staff enrolled in the program offerings. The results in the first stage pointed to five learning strategies. The strategies we refer to as active and disengaged were expected to appear in this analysis. The minimalist strategy is characterised by barely present engagement with the videos and video annotation tool. It was not a frequently adopted strategy, and was mostly present in the first and the last module of both offerings. The remaining two strategies, satisfying module requirements and catching up with the program requirements capture highly valuable patterns of engagement. The former characterises regular engagement with the activities but not beyond the level required by the module. This 
strategy provides a valuable reference of the reasonable dedication and effort that can be assumed by program participants. Fluctuations in the level of presence of this strategy over the course of the semester would point to modules that have unreasonable requirements. In contrast, the same transition in S2 had the opposite effect. The number of teaching staff who were active or satisfying the module requirements increased significantly. Program coordinators may use this information to investigate the differences in the delivery and identify those conditions that are more conducive to engagement with module activities.

Finally, the strategy labelled catching up with the program requirements also provides insight about learners exhibiting erratic patterns of engagement but still committed to the program. Fluctuations in this strategy would point to special circumstances surrounding the delivery, or even increased level of dedication to certain modules. In the context considered in this paper, the presence of this learning strategy is an important indicator for the need for remediation actions, and/or adjustments in the design. Program coordinators may take additional measures to accommodate the needs of teaching staff enrolling in the program or redesign the video annotation activity in the first module altogether.

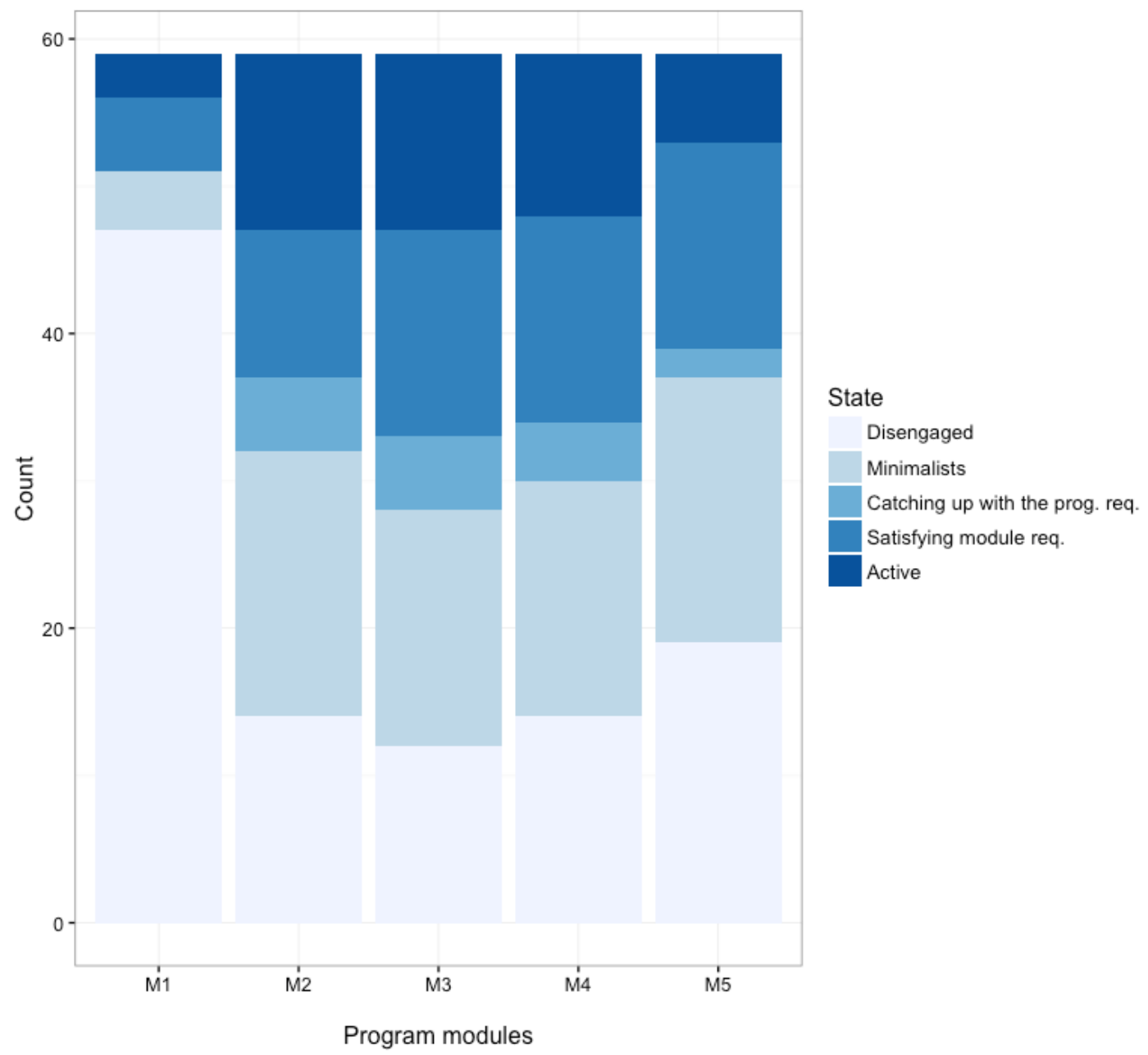

Figure 6. Distribution of teaching staff across the most probable learning strategies in modules (M1-M5), S2 offering.

The results obtained in the first stage of the analysis clearly point to the fact that teaching staff are likely to change their strategies during the learning experience. This finding relates to our second research question which was further explored using the LCA method. The obtained results offer insight into which trajectories or changes in the learning strategies are more likely to occur. The three trajectories identified in S1 (Figure 3) divide users into those that are very likely to sustain their engagement (Trajectory 1), those that remain disengaged (Trajectory 2), and those that fluctuate between minimalists and satisfying the module requirements (Trajectory 3). Program coordinators may use this to contact participants adopting a 
disengaged trajectory to discuss how the program fits with the rest of their responsibilities and assess their motivation and commitment.

The model resulting from LCA does not predict whether and how learners may change learning strategies from one module to the next. Hidden Markov Model has such a predictive capacity, and was used to address our third research question. Figure 5 shows that staff have a strong probability of remaining disengaged, but there is also a small probability for them to adopt a minimalist strategy. On the other hand, the most likely transition from the minimalist and minimalist/disengaged strategies (aside from preserving those strategies) is to become disengaged. Program coordinators can use these probabilities to suggest the actions that will prevent disengagement and lead to the adoption of more engaging strategies.

\section{Conclusion and future directions}

The study showed that participants in a professional development program do not rely on the same learning strategy for the entire program. Rather their strategies tend to change, and in order to explain those changes, researchers need to account for internal and external conditions of the participants' learning context. The Self-regulated learning theoretical framework (Winne \& Hadwin, 1998) allows to establish direct connections between the elements in the learning environment (activities requiring the use of video and annotations) with personal beliefs, goals and strategies. The results described in the study show how learner strategies can be differentiated, and therefore offer the possibility of injecting actions into the environment personalised for every student. Additionally, the strategies learners adopt are mediated by their context, and as a consequence, a detailed characterisation of this element would also enhance the capacity to deploy a more personalised support. This would require the involvement of the learners themselves, and can be done, for example, by conducting interviews with learners to obtain information about their work-related context, and/or asking learners to take field notes to explain the reasons for some of their activities.

Another implication of the presented study is that the peculiarity of work related contexts of lifelong learners in professional development needs to be taken into account in order to optimise their opportunities for deeper engagement with professional development programs. This further implies the need to study pedagogical and other organisational models to find those that would best meet the needs of learners with hectic working life and many activities competing for rather limited time available in their busy schedules.

The limitations of this study points to several directions for further research. A more in-depth study is required to explore an approach to formally transform the insights derived from this study into actionable items. In particular, the information mined from log data could inform the design of a program that provides support actions depending on the detected trajectories. Further, this study only focused on quantitative data, hence, another future direction is to combine quantitative and qualitative information to unpack the reason for the different engagement patterns. Additionally, the quality of the video annotations has not been considered in the study. Future research should consider qualitatively assessing the video annotations alongside quantitative measures used in this study. Finally, the results also point to the need to explore a more holistic view of learner support with actions targeting aspects such as motivation, self-regulation and effective learning.

\section{Acknowledgements}

Support for this publication/activity has been provided by the Australian Government Office for Learning and Teaching. The views expressed in this publication/activity do not necessarily reflect the views of the Australian Government Office for Learning and Teaching.

\section{References}

Akaike, H. (1973). Information theory and an extension of the maximum likelihood principle. In B. N. Petrov, \& F. Cáski (Eds.), Second International Symposium on Information Theory (pp. 267-281). Budapest: Akademiai Kiado

Barab, S. A., Bowdish, B. E., \& Lawless, K. A. (1997). Hypermedia navigation: Profiles of hypermedia users. Educational Technology Research and Development, 45(3), 23-41. 
Bargeron, D., Gupta, A., Grudin, J., \& Sanocki, E. (1999). Annotations for streaming video on the Web: system design and usage studies. Computer Networks, 31(11), 1139-1153.

Blikstein, P., Worsley, M., Piech, C., Sahami, M., Cooper, S., \& Koller, D. (2014). Programming pluralism: Using learning analytics to detect patterns in the learning of computer programming. Journal of the Learning Sciences, 23(4), 561-599. https://doi.org/10.1080/10508406.2014.954750

Brooks, C., Epp, C. D., Logan, G., \& Greer, J. (2011). The who, what, when, and why of lecture Capture. Proceedings of the First International Conference on Learning Analytics and Knowledge, Banff, Canada, 86-92. https://doi.org/10.1145/2090116.2090128

Cleave, J. B., Edelson, D., \& Beckwith, R. (1993, April). A matter of style: An analysis of student interaction with a computer-based learning environment. Proceedings of American Educational Research Association (AERA) Annual Meeting, Atlanta, GA.

Colasante, M. (2011). Using video annotation to reflect on and evaluate physical education pre-service teaching practice. Australasian Journal of Educational Technology, 27(1), 66-88. https://doi.org/10.1145/2090116.2090128

del Valle, R., \& Duffy, T. M. (2009). Online learning: Learner characteristics and their approaches to managing learning. Instructional Science, 37(2), 129-149. https://doi.org/10.1007/s11251-007-9039-0

Gašević, D., Mirriahi, N., \& Dawson, S. (2014). Analytics of the effects of video use and instruction to support reflective learning. Proceedings of the Fourth International Conference on Learning Analytics and Knowledge, Indianapolis, IN, 123-132. https://doi.org/10.1145/2567574.2567590

Giannakos, M. N. (2013). Exploring the video-based learning research: A review of the literature: Colloquium. British Journal of Educational Technology, 44(6), E191-E195. https://doi.org/10.1111/bjet.12070

Giannakos, M. N., Chorianopoulos, K., \& Chrisochoides, N. (2014). Collecting and making sense of video learning analytics. Proceedings of Frontiers in Education Conference, Madrid, Spain, IEEE, 17. https://doi.org/10.1109/FIE.2014.7044485

Giannakos, M. N., Chorianopoulos, K., \& Chrisochoides, N. (2015). Making sense of video analytics: Lessons learned from clickstream interactions, attitudes, and learning outcome in a video-assisted course. The International Review of Research in Open and Distributed Learning, 16(1), 260-283. https://doi.org/10.19173/irrodl.v16i1.1976

Goeze, A., Zottmann, J. M., Vogel, F., Fischer, F., \& Schrader, J. (2014). Getting immersed in teacher and student perspectives? Facilitating analytical competence using video cases in teacher education. Instructional Science, 42(1), 91-114. https://doi.org/10.1007/s11251-013-9304-3

Hadwin, A. F., Nesbit, J. C., Jamieson-Noel, D., Code, J., \& Winne, P. H. (2007). Examining trace data to explore self-regulated learning. Metacognition and Learning, 2(2-3), 107-124. https://doi.org/10.1007/s11409-007-9016-7

Jeong, H., Biswas, G., Johnson, J., \& Howard, L. (2010, June). Analysis of productive learning behaviors in a structured inquiry cycle using hidden Markov models. Paper presented at the International Conference on Educational Data Mining, Pittsburgh, PA.

Koury, K., Hollingsead, C., Fitzgerald, G., Miller, K., Mitchem, K., Tsai, H.-H., \& Zha, S. (2009). Casebased instruction in different delivery contexts: The impact of time in cases. Journal of Interactive Learning Research, 20(4), 445-467.

Kovanović, V., Gašević, D., Joksimović, S., Hatala, M., \& Adesope, O. (2015). Analytics of communities of inquiry: Effects of learning technology use on cognitive presence in asynchronous online discussions. The Internet and Higher Education, 27(October), 74-89. https://doi.org/10.1016/j.iheduc.2015.06.002

Li, N., Kidziński, Ł., Jermann, P., \& Dillenbourg, P. (2015a). MOOC video interaction patterns: What do they tell us? In G. Conole, T. Klobučar, C. Rensing, J. Konert, \& É. Lavoué (Eds.) Design for teaching and learning in a networked world, (pp. 197-210). Toledo, Spain: Springer International Publishing. https://doi.org/10.1007/978-3-319-24258-3_15

Li, N., Kidzinski, L., Jermann, P., \& Dillenbourg, P. (2015b). How do in-video interactions reflect perceived video difficulty? Proceedings of the European MOOCs Stakeholder Summit 2015, Mons, Belgium, 112-121.

Linzer, D. A., \& Lewis, J. B. (2011). poLCA: Polytomous variable latent class analysis. Journal of Statistical Software, 1(10), 1-29. https://doi.org/10.18637/jss.v042.i10

Lockyer, L., Heathcote, E., \& Dawson, S. (2013). Informing pedagogical action: Aligning learning analytics with learning design. American Behavioral Scientist, 57(10), 1439-1459.

https://doi.org/10.1177/0002764213479367 
Lust, G., Elen, J., \& Clarebout, G. (2013a). Regulation of tool-use within a blended course: Student differences and performance effects. Computers \& Education, 60(1), 385-395. https://doi.org/10.1016/j.compedu.2012.09.001

Lust, G., Elen, J., \& Clarebout, G. (2013b). Students' tool-use within a web enhanced course: Explanatory mechanisms of students' tool-use pattern. Computers in Human Behavior, 29(5), 20132021. https://doi.org/10.1016/j.chb.2013.03.014

Lust, G., Vandewaetere, M., Ceulemans, E., Elen, J., \& Clarebout, G. (2011). Tool-use in a blended undergraduate course: In Search of user profiles. Computers \& Education, 57(3), 2135-2144. https://doi.org/10.1016/j.compedu.2011.05.010

McCutcheon, A. L. (1987). Latent class analysis (Vol. 64). Newbury Park: Sage Publications.

Mirriahi, N., \& Dawson, S. (2013). The pairing of lecture recording data with assessment scores: a method of discovering pedagogical impact. In Suthers, D. and Verbert, K. (Eds.) Proceedings of the International Conference on Learning Analytics and Knowledge, Leuven, Belgium, 180-184. https://doi.org/10.1145/2460296.2460331

Mirriahi, N., Liaqat, D., Gašević, D., \& Dawson, S. (2016). Uncovering student learning profiles with a video annotation tool: Reflective learning with and without instructional norms. Educational Technology Research and Development, 64(6) 1083-1106. https://doi.org/10.1007/s11423-016-9449$\underline{2}$

$\mathrm{Mu}, \mathrm{X}$. (2010). Towards effective video annotation: An approach to automatically link notes with video content. Computers \& Education, 55(4), 1752-1763. https://doi.org/10.1016/j.compedu.2010.07.021

Owens, T. (2012). Hitting the nail on the head: the importance of specific staff development for effective blended learning. Innovations in Education and Teaching International, 49(4), 389-400. https://doi.org/10.1080/14703297.2012.728877

Paas, F., Tuovinen, J. E., Tabbers, H., \& Van Gerven, P. W. M. (2003). Cognitive Load Measurement as a Means to Advance Cognitive Load Theory. Educational Psychologist, 38(1), 63-71. https://doi.org/10.1207/s15326985ep3801_8

Pardo, A., Mirriahi, N., Dawson, S., Zhao, Y., Zhao, A., \& Gašević, D. (2015). Identifying learning strategies associated with active use of video annotation software. In G. Siemens, A. Merceron, \& P. Blikstein (Eds), International Conference on Learning Analytics and Knowledge, (pp. 255-259). Poughkeepsie, NY: ACM Press. https://doi.org/10.1145/2723576.2723611

Phillips, R., Maor, D., Preston, G., \& Cumming-Potvin, W. (2012). Exploring learning analytics as indicators of study behaviour. Proceedings of EdMedia: World Conference on Educational Media and Technology, 2861-2867. Denver, CO.

Schwartz, G. (1978). Estimating the dimension of a model. The Annals of Statistics, 6(2), 461-464.

Torrisi-Steele, G., \& Drew, S. (2013). The literature landscape of blended learning in higher education: the need for better understanding of academic blended practice. International Journal for Academic Development, 18(4) 371-383. https://doi.org/10.1080/1360144X.2013.786720

van Gog, T., \& Paas, F. (2012). Cognitive Load Measurement. In N. M. Seel (Ed.), Encyclopedia of the Sciences of Learning (pp. 599-601). Boston, MA: Springer. https://doi.org/ 10.1007/978-1-4419-1428$\underline{6 \quad 412}$

Visser, I., \& Speekenbrink, M. (2010). depmixS4: An R package for hidden Markov models. Journal of Statistical Software, 36(7), 1-21.

Ward, J. H. (1963). Hierarchical grouping to optimize an objective function. Journal of the American Statistical Association, 58(301), 236-244. https://doi.org/10.1080/01621459.1963.10500845

Weinstein, C. E., Husman, J., \& Dierking, D. R. (2000). Self-regulation interventions with a focus on learning strategies. In M. Z. Boekaerts, M. R. Pintrich, \& M. Zeidner (Eds.), Handbook of Selfregulation, (pp. 727-768) San Diego, CA: Academic Press.

Winne, P. H. (2006). How software technologies can improve research on learning and bolster school reform. Educational Psychologist, 41(1), 5-17. https://doi.org/10.1207/s15326985ep4101_3

Winne, P. H. (2013). Learning strategies, study skills, and self-regulated learning in postsecondary education. In M. B. Paulsen (Ed.), Higher education: Handbook of theory and research (pp. 377-403). Netherlands: Springer Science+Business Media. https://doi.org/10.1007/978-94-007-5836-0_8

Winne, P. H., \& Hadwin, A. F. (1998). Studying as self-regulated learning. In D. J. Hacker, J. Dunlosky, \& A. C. Graesser (Eds.), Metacognition in educational theory and practice (pp. 227-304). Mahwah, NJ: Lawrence Erlbaum Associates.

Winne, P. H., \& Jamieson-Noel, D. (2002). Exploring students' calibration of self reports about study tactics and achievement. Contemporary Educational Psychology, 27(4), 551-572. 
Yousef, A. M. F., Chatti, M. A., \& Schroeder, U. (2014). Video-based learning: A critical analysis of the research published in 2003-2013 and future visions. Proceedings of eLmL 2014, The Sixth International Conference on Mobile, Hybrid, and On-line Learning, Barcelona, 112-119.

Zimmerman, B. J. (2008). Investigating self-regulation and motivation: Historical background, methodological developments, and future prospects. American Educational Research Journal, 45(1), 166-183. https://doi.org/10.3102/000283120

Corresponding author: Negin Mirriahi, negin.mirriahi@unisa.edu.au

Australasian Journal of Educational Technology (c) 2018.

Please cite as: Mirriahi, N., Jovanovic, J., Dawson, S., Gašević, D., \& Pardo, A. (2018). Identifying engagement patterns with video annotation activities: A case study in professional development. Australasian Journal of Educational Technology, 34(1), 57-72. https://doi.org/10.14742/ajet.3207 